\title{
Supramolecular structure of dietary fat in early life modulates expression of markers for mitochondrial content and capacity in adipose tissue of adult mice
}

Andrea Kodde ${ }^{1^{*}} \mathbb{D}$, Eline M. van der Beek ${ }^{1,2}$, Esther Phielix $^{3}$, Eefje Engels ${ }^{1}$, Lidewij Schipper $^{1}$ and Annemarie Oosting ${ }^{1}$

\begin{abstract}
Background: Previous studies have shown that early life nutrition can modulate the development of white adipose tissue and thereby affect the risk on obesity and metabolic disease later in life. For instance, postnatal feeding with a concept infant milk formula with large, phospholipid coated lipid droplets (Concept, Nuturis ${ }^{\circledast}$ ), resulted in reduced adiposity in adult mice. The present study investigated whether differences in cell energy metabolism, using markers of mitochondrial content and capacity, may contribute to the observed effects.

Methods: C57BI/6j male mice were exposed to a rodent diet containing the Concept (Concept) or standard (CTRL) infant milk formula from postnatal day 16 until postnatal day 42, followed by a western style diet challenge until postnatal day 98. Markers for mitochondrial content and capacity were analyzed in retroperitoneal white adipose tissue and gene expression of metabolic markers was measured in both retroperitoneal white adipose tissue and muscle tibialis (M. tibialis) at postnatal day 98.

Results: In retroperitoneal white adipose tissue, the Concept group showed higher citrate synthase activity and mitochondrial DNA expression compared to the CTRL group $(p<0.05)$. In addition, protein expression of mitochondrial cytochrome c oxidase subunit I of the oxidative phosphorylation pathway/cascade was increased in the Concept group compared to CTRL $(p<0.05)$. In the M. tibialis, gene expression of uncoupling protein 3 was higher in the Concept compared to the CTRL group. Other gene and protein expression markers for mitochondrial oxidative capacity were not different between groups.

Conclusion: Postnatal feeding with large, phospholipid coated lipid droplets generating a different supramolecular structure of dietary lipids enhances adult gene and protein expression of specific mitochondrial oxidative capacity markers, indicative of increased substrate oxidation in white adipose tissue and skeletal muscle. Although functional mitochondrial capacity was not measured, these results may suggest that adaptations in mitochondrial function via early feeding with a more physiological structure of dietary lipids, could underlie the observed beneficial effects on later life adiposity.
\end{abstract}

Keywords: Postnatal programming, White adipose tissue, Mitochondrial content, Oxidative capacity, Lipid droplet structure

\footnotetext{
* Correspondence: andrea.kodde@danone.com

${ }^{1}$ Earl Life Nutrition Division, Nutricia Research, P.O. Box 801413508 TC

Utrecht, The Netherlands

Full list of author information is available at the end of the article
} 


\section{Background}

Obesity is a global health threat and increasingly emerges at a young age [1]. Early onset obesity is associated with metabolic disease, including diabetes and cardiovascular disease [1]. Increased energy intake and decreased physical activity are well-established lifestyle risk factors in obesity [2]. When energy intake exceeds energy expenditure, the surplus energy is stored in white adipose tissue (WAT) resulting in increased adipocyte size (hypertrophy) and eventually increased adipocyte numbers (hyperplasia) [3-5]. When WAT fails to store the excessive energy, fat accumulates ectopically in the liver and skeletal muscle, an important step in the development of type 2 diabetes (T2D) [6].

A mismatch between storage of fat in the form of triglycerides and oxidation of fatty acids (FA) in the mitochondria is an important feature in both obesity and T2D [7]. Moreover, compromised mitochondrial function in skeletal muscle [8-10], as well as in liver and WAT $[11,12]$ evaluated using functional measurements, is a well know feature seen in obesity and T2D. In line with these observations, gene expression of a subset of genes coding for mitochondrial proteins (i.e. pyruvate dehydrogenase, cytochrome C, carnitinepalmitoyltransferase I (Cpt1) and the uncoupling proteins (Ucps)) were lower in WAT of transgenic diabetic mice models and in high fat diet (HFD)-induced insulin-resistant mice $[11,13]$. Taken together, these data strongly indicate a pivotal role of mitochondrial energy metabolism in WAT in obesity and T2D.

The development of WAT starts in the third trimester of gestation and continues throughout childhood and adolescence [3]. Nutrition (excess or scarcity) during pregnancy and infancy has a substantial effect on adipose tissue development. For instance, maternal exposure to a HFD during pregnancy and lactation resulted in increased adiposity in rodent offspring $[14,15]$. This indicates that the nutrition in early life programs adiposity in adulthood.

Observational studies have demonstrated a moderate but consistent association between breastfeeding (duration) and reduced prevalence of later life overweight and obesity [16, 17]. Studies from our lab suggest that the supramolecular structure of lipids in milk may contribute to these beneficial effects [18-20]. We have shown that early postnatal exposure to a diet containing a concept IMF with large lipid droplets coated by phospholipids (Concept, Nuturis ${ }^{\oplus}$ ) reduced body fat accumulation in adolescence and early adulthood in a mouse model for nutritional programming $[18,19]$. The reduced adult fat mass was associated with reduced adipocyte size and altered adipocyte function as well as improved metabolic health [19]. This provides clear evidence that early life dietary lipid supramolecular structure may effectively program metabolic health later in life.

Nutrition during fetal and early postnatal life has been shown to affect mitochondrial content and oxidative capacity throughout life [21-24]. For instance, hepatic enzyme activity of the mitochondrial oxidative phosphorylation was decreased in offspring of dams fed a HFD during pregnancy and lactation [21]. Thus, differences in mitochondrial development and function may underlie programming of adiposity and metabolic health later in life.

In the current study, we investigate if postnatal exposure to large, phospholipid coated lipid droplets affects markers for mitochondrial content and oxidative capacity in WAT. We hypothesize that the supramolecular lipid structure affects the expression of genes involved in the energy utilization in both WAT and skeletal muscle and the expression of markers for mitochondrial content and oxidative capacity in WAT.

\section{Methods}

Animals and study design All experimental procedures were approved by an external, independent Animal Experimental Committee (DEC consult, Soest, The Netherlands) and complied with the principles of good laboratory animal care following the EU-directive for the protection of animals used for scientific purposes. C57Bl/6jOlaHsd mice (Harlan, Horst, The Netherlands) were kept at the animal facility of Wageningen University and Research Centre under a $12 \mathrm{~h}$ light - $12 \mathrm{~h}$ dark cycle (lights on at 06:00 h). Room temperature and humidity were kept at a constant level $\left(21 \pm 2{ }^{\circ} \mathrm{C}\right.$ and $50 \pm 5 \%$ respectively) and standard cage enrichment was added to allow mice to build a nest. Food and water were available ad libitum during the entire experimental period, except for an $8 \mathrm{~h}$ fastening period before dissection. Breeders were time-mated and female mice were provided with the American Institute of Nutrition-93G synthetic diet (AIN93G) [25] during pregnancy and lactation. At postnatal day 2 (PN2), litters were randomly cross-fostered to reduce between-litter variability and avoid litter effects, and culled to four male and two female pups per dam. On PN16, litters were randomly assigned to either the $i$. control (CTRL), ii. Concept or iii. Non-challenged reference group (REF), resulting in $n=12$ male pups per experimental group (3 litters per group). The Concept group was fed the Concept-IMF based diet and CTRL and REF group were fed a controlIMF based diet from PN16 until PN42 (Fig. 1). Male littermates were housed in pairs after weaning at PN21 and continued their respective IMF-based diets. From PN42 until PN98, Concept and CTRL group were challenged with a western style diet containing $20 \mathrm{w} / \mathrm{w} \%$ fat (WSD, Fig. 1). The REF group was fed AIN93M [25] 


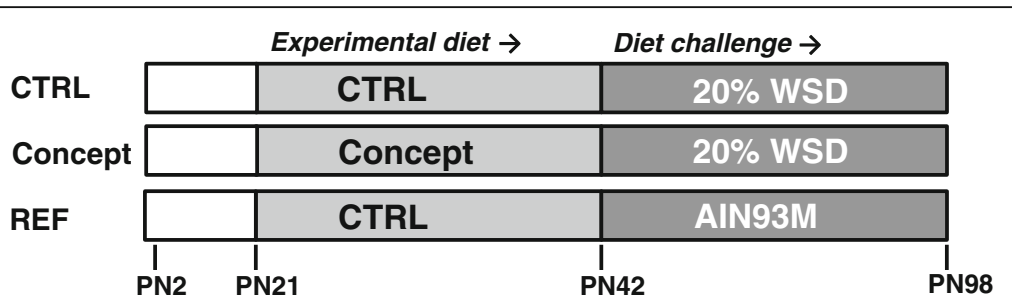

Fig. 1 Study design; WSD: western style diet; PN: postnatal day

from PN42 until PN98 and was included as a nonchallenged reference to compare to the effects of the WSD challenge (CTRL group). Body weight was measured twice a week as of the period of weaning and body composition was monitored by DEXA using a PIXImus imager (GE Lunar, Madison, WI, USA) under general anesthesia (isoflurane $/ \mathrm{N}_{2} \mathrm{O} / \mathrm{O}_{2}$ ). At PN98, mice were fasted for $8 \mathrm{~h}$, subjected to terminal anesthesia (isoflurane $/ \mathrm{N}_{2} \mathrm{O} / \mathrm{O}_{2}$ ) and dissected. M.tibialis and retroperitoneal (RP) WAT depots (as proxy for visceral WAT) were weighed, snap frozen and stored at $-80{ }^{\circ} \mathrm{C}$. Epididymal (EPI) WAT depots were weighed and immediately used for fat cell analysis, as RP WAT depots were too small to use for all analyses.

IMF production The CTRL IMF was produced according to a Standard stage 1 IMF recipe and processing procedure (Nutricia Research, Utrecht, the Netherlands). For the Concept IMF powder $0.5 \mathrm{~g} / \mathrm{l}$ phospholipids of bovine milk origin (SM2, Corman Food industry, Goé, Belgium) was added and processing procedure was adjusted as described previously $[19,26]$. The mode diameter of the lipid droplets in the Concept IMF was $2.924 \mu \mathrm{m}$ versus $0.267 \mu \mathrm{m}$ in the CTRL IMF.

Experimental diets Control and Concept IMF-based diets were semi-synthetic and contained $28.2 \mathrm{w} / \mathrm{w} \%$ control or Concept IMF powder, respectively. The diets were complemented with protein and carbohydrates to establish macronutrient composition appropriate for normal growth and development in rodents and match a macronutrient composition according to AIN93G standards (Table 1). The macronutrient composition of CTRL and Concept diet was similar except for phospholipid (0.2 versus $1.44 \mathrm{~g} / \mathrm{kg}$, respectively) content. The moderate WSD consisted of $20 \%(\mathrm{w} / \mathrm{w})$ fat of which $17 \%(\mathrm{w} / \mathrm{w})$ lard, 3\% $(w / \mathrm{w})$ soy oil and $0.1 \%(w / \mathrm{w})$ cholesterol (Table 1$)$.

Fat cell analysis Fresh EPI WAT depots were used to determine cell size distribution according to the optical method of DiGirolamo and Fine [27] and Hirsch and Gallian [28] as described in detail previously [19].

Table 1 Composition of the early programming diets and the WSD diet

\begin{tabular}{|c|c|c|c|c|}
\hline \multirow[t]{2}{*}{ Diet } & & \multicolumn{2}{|c|}{ Programming diet } & \multirow[t]{2}{*}{ WSD } \\
\hline & & CTRL & Concept & \\
\hline Carbohydrates & $(\mathrm{g} / \mathrm{kg})$ & 646 & 646 & 520 \\
\hline Sugars $^{a}$ & $(g / k g)$ & 236 & 236 & 70 \\
\hline Polysaccharides $^{b}$ & $(g / k g)$ & 410 & 410 & 450 \\
\hline Protein & $(g / k g)$ & 179 & 179 & 180 \\
\hline Fat & $(g / k g)$ & 70 & 70 & 200 \\
\hline Saturated Fatty Acids (SFA) & $(\mathrm{g} / \mathrm{kg})$ & 30 & 30 & 81,2 \\
\hline Mono Unsaturated Fatty Acids (MUFA) & $(g / k g)$ & 28 & 28 & 84,9 \\
\hline Poly Unsaturated Fatty Acids (PUFA) & $(\mathrm{g} / \mathrm{kg})$ & 12 & 12 & 32,7 \\
\hline LA/ALA ratio & & 5.5 & 5.5 & 14,9 \\
\hline Phospholipids ${ }^{c}$ & $(\mathrm{~g} / \mathrm{kg})$ & 0.2 & 1.44 & - \\
\hline Cholesterol & $(\mathrm{g} / \mathrm{kg})$ & - & - & 1.0 \\
\hline Fiber & $(\mathrm{g} / \mathrm{kg})$ & 47.5 & 47.5 & 50 \\
\hline Vitamin \& mineral mix & $(\mathrm{g} / \mathrm{kg})$ & 45 & 45 & 45 \\
\hline
\end{tabular}

${ }^{\mathrm{a}}$ Total sugars, including lactose, glucose and sucrose

bIncluding starch and maltodexintrin

'Phospholipids derived from bovine milk, as a and b 
Gene expression RNA from RP WAT and m.tibialis were isolated using $\mathrm{Trizol}^{\circ} /$ chloroform (Invitrogen, Breda, The Netherlands) and purified with a RNeasy Mini Kit (Qiagen Benelux b.v., Zwijndrecht, The Netherlands) including a DNase treatment with a RNase-free DNase Set (Qiagen Benelux b.v., Zwijndrecht, The Netherlands) as previously described [29]. Quantity and quality of the RNA samples was analysed with the Nanodrop 2000 (Thermo Scienctific, Breda, The Netherlands) and the Bioanalyzer (Agilent, Santa Clara, USA). Samples with a RIN below 8.0 and a 260/ 280 ratio below 1.9 were excluded from analyses. cDNA was synthesized with the iScript ${ }^{\text {tu }}$ cDNA synthese kit (Bio-Rad, Veenendaal, The Netherlands) according to manufacturer instructions. 9,4 and 25 ng cDNA was used as input for each Q-PCR reaction of the RP WAT and m.tibialis samples, respectively. $5 \times$ Hot FirePol Evagreen $^{\circ}$ qPCR mix Plus (Bio-Connect, Huissen, The
Netherlands) was used according to manufacturer instructions and qPCR was performed with a 7900HT Fast Real Time PCR System (Applied Biosystems, Bleiswijk, The Netherlands). Acetyl-CoA carboxylase 1 (Acc1), adipose triglyceride lipase (Atgl), fatty acid transfer protein/ cluster of differentiation 36 (Cd36), Cell death-inducing DNA fragmentation factor, alpha subunit-like effector A (Cidea), Cpt1 $\alpha$, fatty acid binding protein 4 (Fabp 4 ), fatty acid synthase (Fasn), fatty acid transfer protein 1 (Fatp1), glucose transporter 4 (Glut4), glycerol-3-phosphate acyltransferase (Gpat), hexokinase II (HkII), hormone sensitive lipase $(H s l)$, lipoprotein lipase $(L p l)$, pyruvate dehydrogenase kinase $4(P d k 4)$, peroxisome proliferator activated receptor $\alpha$ (Ppar $\alpha)$, stearoyl-Coenzyme A desaturase $1(S c d 1)$, sterol regulatory element binding protein 1c (Srebp1c), Ucp1 and Ucp3 mRNA expression

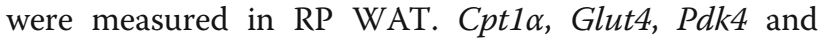
Ucp3 gene expression were measured in m.tibialis. See

Table 2 Primer sequences

\begin{tabular}{|c|c|c|c|}
\hline Gene name & NCBI Reference number & Forward primer & Reverse primer \\
\hline \multicolumn{4}{|c|}{ Genes of interest: } \\
\hline Accl & NM_133360.2 & tggtgcagaggtaccgaagtg & cgtagtggecgttctgaaact \\
\hline Atgl & NM_025802.3 & atccgcttgttggagtggct & ctcttggccctcatcaccag \\
\hline Cd36 & NM_001159558.1 & attgcgacatgattaatggcac & gatagacctgcaaatgtcagaggaa \\
\hline Cidea & NM_007702.2 & aggccgtgttaaggaatctg & cccagtactcggagcatgta \\
\hline Cpt1a & NM_013495.2 & gcccatgttgtacagcttcc & tcatcagtggectcacagac \\
\hline Fabp4 & NM_024406.2 & tcataaccctagatggcggg & ttccaccaccagcttgtcac \\
\hline Fas & NM_007988.3 & gctgggctctatggattaccc & ggtccattgtgtgtgectgc \\
\hline Fatp1 & NM_011977.3 & attgtggtgcacagcaggtacta & tggtagagtggcaggcagtca \\
\hline Glut4 & NM_009204.2 & cggatgctatgggtccttacg & aacgtccggectctggttt \\
\hline Gpat & NM_008149.3 & tgtccacaacttcagcggtc & cagcgtacacggcaacgtt \\
\hline$H k \|$ & NM_013820.3 & aagacgataaggacggaattcaga & gagcgcgtggacacaatct \\
\hline Hsl & NM_010719.5 & cctgcttggttcaactggaga & tcactccataggctgctgcc \\
\hline$L p l$ & NM_008509.2 & cacttgtcatctcattcctggatt & ccgttaccgtccatccatg \\
\hline$P d k 4$ & NM_013743.2 & aagagctggtatatccagagectg & ttgaccagcgtgtctacaaactc \\
\hline Ppara & NM_011144.6 & agtgccctgaacatcgagtgt & ttcgccgaaagaagccctta \\
\hline Scdl & NM_009127.4 & cacctgcctcttcgggatttt & gcagccgtgccttgtaagttc \\
\hline Srebplc & NM_011480.3 & ccggctattccgtgaacatc & caagggcatctgagaactccc \\
\hline Ucp 1 & & assay ID qMmuCID0005832 & \\
\hline Ucp3 & NM_009464.3 & aacgctcccctaggcaggta & gcagaaaggagggcacaaatc \\
\hline \multicolumn{4}{|c|}{ Reference genes: } \\
\hline Canx & NM_007597.3 & agagctcagcctggatcaattc & ttgtagtcctctccacacttatctgg \\
\hline Rpl19 & NM_009078.2 & ttgcctctagtgtcctccgc & cttcctgatctgctgacggg \\
\hline Rps29 & NM_009093.2 & agtcacccacggaagttcgg & gtccaacttaatgaagcctatgtcctt \\
\hline 18SrRNA & NR_003278.1 & cgattccgtgggtggtggtg & catgccagagtctcgttcgttatc \\
\hline \multicolumn{4}{|l|}{ DNA primers } \\
\hline $\mathrm{Nd1}$ & NC_005089.1 & accaatacgecctttaacaac & aatgggtgtggtattggtagg \\
\hline$L p l$ & NM_008509 & tcctgatgacgctgattttg & atgtcaacatgccctactgg \\
\hline
\end{tabular}


Table 2 for the complete list of primers. RP WAT qPCR data was normalized using the method of relative normalization as described by Hellemans et al. [30] and performed with qbase $^{+}$(Biogazelle, Genth, Belgium). In short, gene expression of genes of interest was analyzed relative to mean expression of multiple reference genes and scaled to the expression of the CTRL group. Four reference genes were used for the RP WAT: ribosomal protein L19 (Rpl19) and S29 (Rps29), 18S ribosomal RNA (18SrRNA) and Calnexin (Canx). Rpl19, Rps29 and 18SrRNA were used as reference genes for the M. tibialis.

Enzyme activity Citrate synthase (CS) and Hydroxyacyl-CoA dehydrogenase (HAD) activity were measured as described elsewhere [31]. In short, RP WAT samples were homogenized and dissolved in sucrose-Tris-EDTA buffer $(250 \mathrm{mM}$ Sucrose, $10 \mathrm{mM}$ Tris, 2 mM EDTA, pH 7.4). CS activity was analyzed by adding sample to reaction reagent $(100 \mathrm{mM}$ Tris, $100 \mu \mathrm{M}$ DTNB, $50 \mu \mathrm{M}$ acetyl-CoA, pH 8.0) and start reagent (50 $\mathrm{mM}$ Oxaloacetic acid), in the proportion 1:50:1, followed by a kinetic reading $\left(421 \mathrm{~nm}, 37^{\circ} \mathrm{C}\right)$. HAD activity was analyzed by adding sample to reaction reagent (100 mM tetra-sodiumpyrophosphate, $250 \mu \mathrm{M}$ $\mathrm{NADH}, \mathrm{pH} 7.3$ ) and start reagent (5 mM AcetoacetylCoA), in the proportion 1:10:1, followed by a kinetic reading $\left(340 \mathrm{~nm}, 37^{\circ} \mathrm{C}\right)$.

OXPHOS protein expression Protein expression of 5 subunits of the oxidative phosphorylation (OXPHOS) was measured as previously described [32]. Briefly, RP WAT samples were homogenized and dissolved in Radio-Immunoprecipitation Assay buffer (RIPA, Fisher Scientific, Landsmeer, The Netherlands) with protease inhibitor cocktail (Roche diagnostics, Almere, the Netherlands). Per sample $15 \mu \mathrm{g}$ was used for gel electrophoreses with a $4-15 \%$ gradient and transferred to a PVDF membrane with a Trans-Blot ${ }^{\circ}$ Turbo $^{\mathrm{Tm}}$ Blotting System using the Trans-Blot ${ }^{\oplus}$ Turbo $^{\mathrm{Tm}}$ Midi PVDF Transfer pack (Bio-Rad, Veenendaal, The Netherlands). OXPHOS protein expression was determined using the Mito-Profile ${ }^{\oplus}$ Total OXPHOS rodent western blot antibody cocktail (Abcam, Cambridge, UK) with ECL anti mouse IgG (Fisher Scientific, Landsmeer, The Netherlands) as a secondary antibody. Protein expression was detected with the ChemiDoc ${ }^{\text {тм }}$ XRS, analyzed by Quantity One (Biorad, Veenendaal, The Netherlands) and adjusted for total protein levels per lane, using coomassie brilliant blue staining.

Mitochondrial DNA content Nuclear and mitochondrial DNA (mtDNA) was isolated from RP WAT with the QIAamp DNA micro kit (Qiagen Benelux b.v., Zwijndrecht, The Netherlands), according to manufacturers protocol. DNA quantity was determined with a Nanodrop 2000 (Thermo Scienctific, Breda, The Netherlands). Relative mitochondrial DNA expression was determined as previously described [33]. Briefly, $135 \mathrm{ng}$ input DNA was used for each qPCR reaction, with NADH dehydrogenase $1(N d 1)$ as a marker for mitochondrial DNA and $L p l$ to normalize for nuclear DNA. Primers sequences are shown in Table 2. Data were analyzed using qbase ${ }^{+}$(Biogazelle, Genth, Belgium).

Statistical analysis Statistical analyses were performed using SPSS ${ }^{\bullet} 19.0$ (SPSS Benelux, Gorinchem, The Netherlands). The primary focus for the study was to assess the difference between the Concept and the CTRL group, which was tested using a TTest. The latter will give insight into the window of opportunity to be influenced by our nutritional intervention as well as to understand the direction of change. The effect of the WSD challenge was tested separately using a TTest. Concept and REF group were not directly compared based on the differences in dietary exposure both in early postnatal life (Concept vs. Control) and also later life (WSD vs. AIN). Gaussian distribution was tested with a KolmogorovSmirnov test. Differences in adipocyte size distribution were analyzed with a Mann-Whitney. Correlations between parameters were analyzed using a Pearson test. Data are presented as mean $+\mathrm{SD}$, except for the qPCR data which are presented as mean relative expression (scaled to the average expression) with 95\% confidence intervals. Differences were considered significant at $p<0.05$ and tendencies reported when $0.05<p<0.1$.

\section{Results}

Effect of the western-style diet challenge during adolescence and adulthood Body composition

As a consequence of the WSD challenge, body weight, fat mass, EPI and RP WAT weight were increased in the CTRL compared to the non-challenged REF group. Lean body mass was slightly higher although no significant effect on $M$. tibialis weight was found (Table 3). These data are discussed elsewhere [20].

\section{Adipocyte size and number in EPI WAT}

The WSD challenge resulted in a $44 \%$ increase in average EPI adipocyte size in the CTRL group compared to the non-challenged REF group ( $p<0.001$; Table 3$)$. Furthermore, a shift was seen in adipocyte size distribution towards larger cells in the CTRL group (Fig. 2). Percentages of cells in the size categories between 30 and $90 \mu \mathrm{m}$ were lower for the CTRL group while higher in the size categories between 100 and $240(p<0.05)$. EPI adipocyte numbers tended to be lower in the CTRL group $(p=0.07$; Table 3$)$. 
Table 3 Body composition, organ weight and EPI WAT adipocyte characteristics (mean adipocyte size and number)

\begin{tabular}{|c|c|c|c|}
\hline Diet group & $\operatorname{REF}(n=12)$ & $\mathrm{CTRL}(n=12)$ & Concept $(n=12)$ \\
\hline Body weight (g) & 28.5 (SD 1.8) & 31.7 (SD 2.5) ${ }^{\# \#}$ & $29.2($ SD 2.4)* \\
\hline Lean body mass (g) & 23.5 (SD 1.4) & $24.4(S D$ 1.3) $†$ & $23.5(\mathrm{SD} 1.8)$ \\
\hline Fat mass (g) & 4.9 (SD 1.1) & 7.5 (SD 1.8) ${ }^{\# \# \#}$ & $5.8(S D ~ 1.6) *$ \\
\hline RP WAT (g) & 0.14 (SD 0.06) & 0.31 (SD 0.10) & $0.20($ SD 0.10) * \\
\hline EPI WAT (g) & 0.48 (SD 0.15) & 1.00 (SD 0.29) \#\#\# & $0.63(\mathrm{SD} 0.27) * *$ \\
\hline M. tibialis (g) & 0.05 (SD 0.00) & 0.05 (SD 0.01) & 0.05 (SD 0.01) \\
\hline Adipocyte characteristics: & $\operatorname{REF}(n=11)$ & CTRL $(n=10)$ & Concept $(n=9)$ \\
\hline Mean adipocyte size $(\mu \mathrm{m})$ & 74.4 (SD 10.3) & 107.1 (SD 15.7) \#\#\# & $81.3($ SD 14.0)** \\
\hline Adipocyte number $\left(\times 10^{6}\right)$ & 7.44 (SD 2.44) & $5.79(\mathrm{SD} 1.07)+$ & 7.55 (SD 3.67) \\
\hline
\end{tabular}

Data expressed as mean plus SD. Early diet and WSD challenge effects were analyzed separately. Difference between Concept and REF groups were not tested, as groups were fed different postnatal and adult diets. \#\#: $p<0.01$; \#\#\#: $p<0.001 ;$ †: $p=0.05-0.10$, CTRL different from REF group; *: $p<0.05 ;{ }^{* *}: p<0.01$, Concept different from CTRL group

\section{Markers for mitochondrial content and $\beta$-oxidation in the WAT}

Mitochondrial content of the RP depot, as measured by CS activity and relative mtDNA content, was significantly lower in the CTRL group compared to the REF group ( $p<0.05$; Fig. 3a and b). FA oxidation of the RP WAT, reflected by HAD activity, was similar in both groups (Fig. 3c).

\section{OXPHOS protein expression in the WAT}

No effect of the WSD challenge was shown on the protein expression of OXPHOS complexes I (NDUFB8; Fig. 4a), II (SDHB; Fig. 4b), III (UQCRC2; Fig. 4c) and IV (MTCOI; Fig. 4d) in the RP depot. Expression levels of the ATP synthase subunit of complex V (ATP5A) tended to be increased in the CTRL group as a consequence of the WSD challenge ( $p=0.08$; Fig. $4 \mathrm{e})$. OXPHOS protein expression was lower for all complexes in the CTRL compared to the REF group when corrected for CS activity (Fig. 5a-e).

\section{Gene expression profiles RP WAT}

Expression levels of the lipogenic genes Gpat and Srebp1c in RP WAT were lower in the CTRL compared to the REF group as were the expression levels of the lipogenic enzymes Acc1, Fas and Scd1 ( $p<0.05$ for all parameters; Table 4). Gene expression of the lipolytic enzyme Atgl tended to be lower in the CTRL group $(p=0.09)$. Expression of $H s l$ was similar in both groups. Expression levels of $P d k 4$ and HkII, both part of the glucose oxidative pathway, were significantly lower in the CTRL compared to the REF group as were the levels of the glucose transporter Glut4 ( $p<0.05$ for all parameters; Table 4). The CTRL group showed elevated expression levels of the FA transporters $C p t 1 \alpha$ and Cd36 and lower Fatp1 and Fabp4 levels compared to the non-challenged REF group $(p<0.05$ for all parameters). No difference was observed in expression levels of LPL (Table 4). Ppara expression levels were lower in the CTRL group compared to the REF group $(p<0.001)$, as was the UCP3 gene expression $(p<0.01$; Table 4$)$. Expression of WAT browning markers Ucp1 and Cidea were

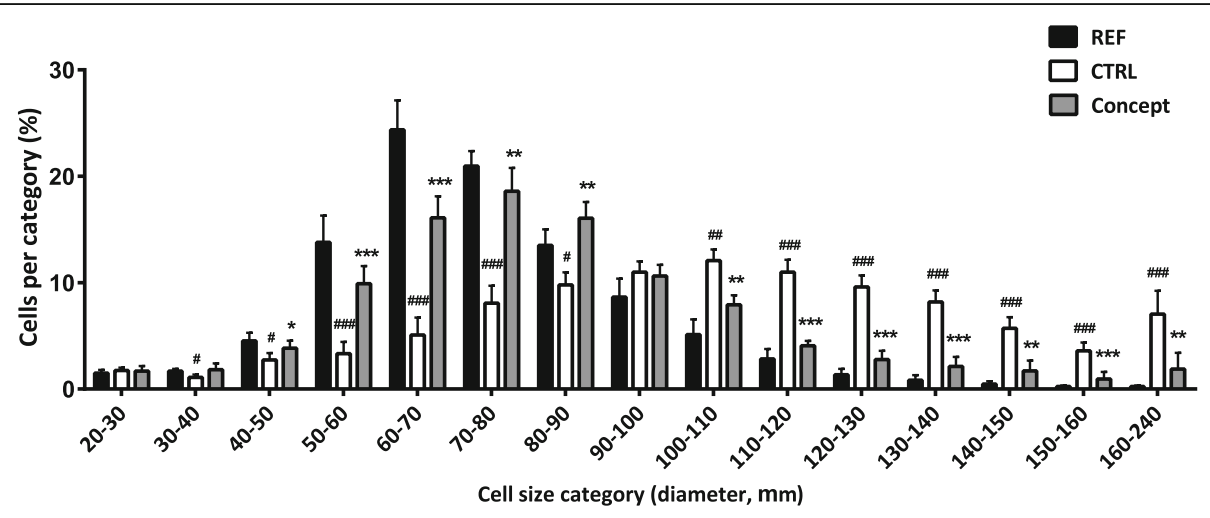

Fig. 2 Effect of postnatal Concept diet on adult adipocyte size distribution of epididymal white adipose tissue $(n=10, n=9$ and $n=11$ for CTRL, Concept and REF group respectively). Early diet and WSD challenge effects were analyzed separately. Difference between Concept and REF group not tested, as groups were fed different postnatal and adult diets. \#: $p<0.05$; \#\#: $p<0.01$; \#\#\#: $p<0.001$, CTRL different from REF group; *: $p<0.05 ;{ }^{* *}: p<0.01$; $^{* *}: p<0.001$ Concept different from CTRL group 


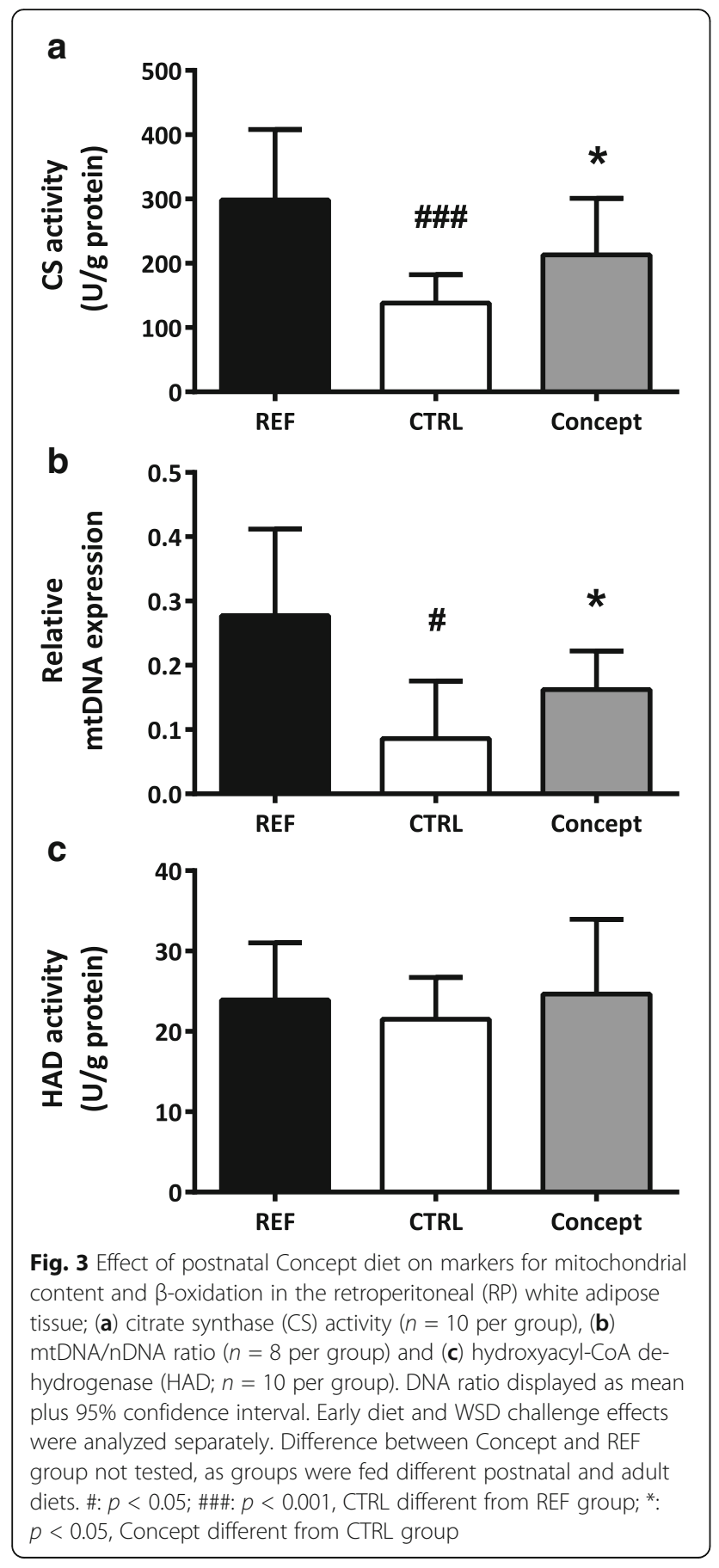

decreased in the CTRL compared to the Ref group $(p<0.001$; Table 4$)$.

\section{Gene expression profiles in skeletal muscle}

The CTRL group showed lower Ucp3 expression levels in the $m$. tibialis compared to the REF group $(p<0.01)$. Expression profiles of Pdk4,Cpt1 $\alpha$ and Glut4 were not affected by the adult WSD challenge (Table 4).
Programming effect of concept IMF diet Body composition

In accordance to previous findings $[18,19]$, body weight, fat mass, EPI and RP WAT weight were reduced in the Concept compared to the CTRL group. Lean body mass and $m$. tibialis weight were similar in both groups (Table 3 ). These data are part of a more extensive study concerning the potential protective effects of structural aspect of lipids in human milk against later life obesity and therefore reported in a separate paper [20].

\section{Adipocyte size and number in EPI WAT}

Average EPI adipocyte size (Table 3) was 24\% lower in the Concept group compared to the CTRL group $(p<0.01)$. This was confirmed by a shift in adipocyte size distribution towards smaller cells in the Concept compared to the CTRL group (Fig. 2). Most striking differences were found in size categories between 50 and $90 \mu \mathrm{m}$ with more cells for the Concept compared to the CTRL group $(p<0.05)$. In addition, the Concept group showed fewer cells in the size categories between 100 and $240 \mu \mathrm{m}(p<0.01)$ compared to the CTRL group. EPI adipocyte numbers were similar in both groups (Table 3).

\section{Markers for mitochondrial content and $\beta$-oxidation in WAT}

In the RP depot mitochondrial content, as measured by CS activity and relative mtDNA expression, was significant higher in the Concept compared to the CTRL group ( $p<0.05$; Fig. 3a and b respectively). Groups had similar HAD activity, which reflects FA oxidation (Fig. 3c).

\section{OXPHOS protein expression in WAT}

Mitochondrial cytochrome c oxidase I subunit (subunit of complex IV, MTCOI) expression levels were significantly higher in the Concept group compared to the CTRL group ( $p<0.05$; Fig. $4 d)$. Protein expression of complex I (NDUFB8; Fig. 4a) II (SDHB; Fig. 4b), III (UQCRC2, Fig. 4c) and V (ATP5A; Fig. 4e) did not significantly differ between the two groups. OXPHOS protein expression was not significantly lower in the Concept compared to the CTRL group when corrected for CS activity (Fig. 5a-e).

\section{Gene expression profiles in RP WAT}

RP WAT expression levels of lipogenic genes Gpat, Srebp1c, Acc1, Fas and Scd1 were similar in the Concept and the CTRL group as were the expression levels of lipolytic enzymes Atgl and $\mathrm{Hsl}$ (Table 4).

Glucose oxidative pathways (PDK4 and HKII) were unaffected by the early diet (Table 4). The $C d 36$ expression levels of the Concept group tended to be lower compared to the CTRL group $(p=0.09)$, but expression

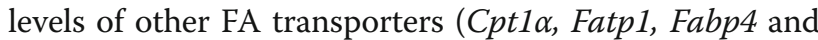



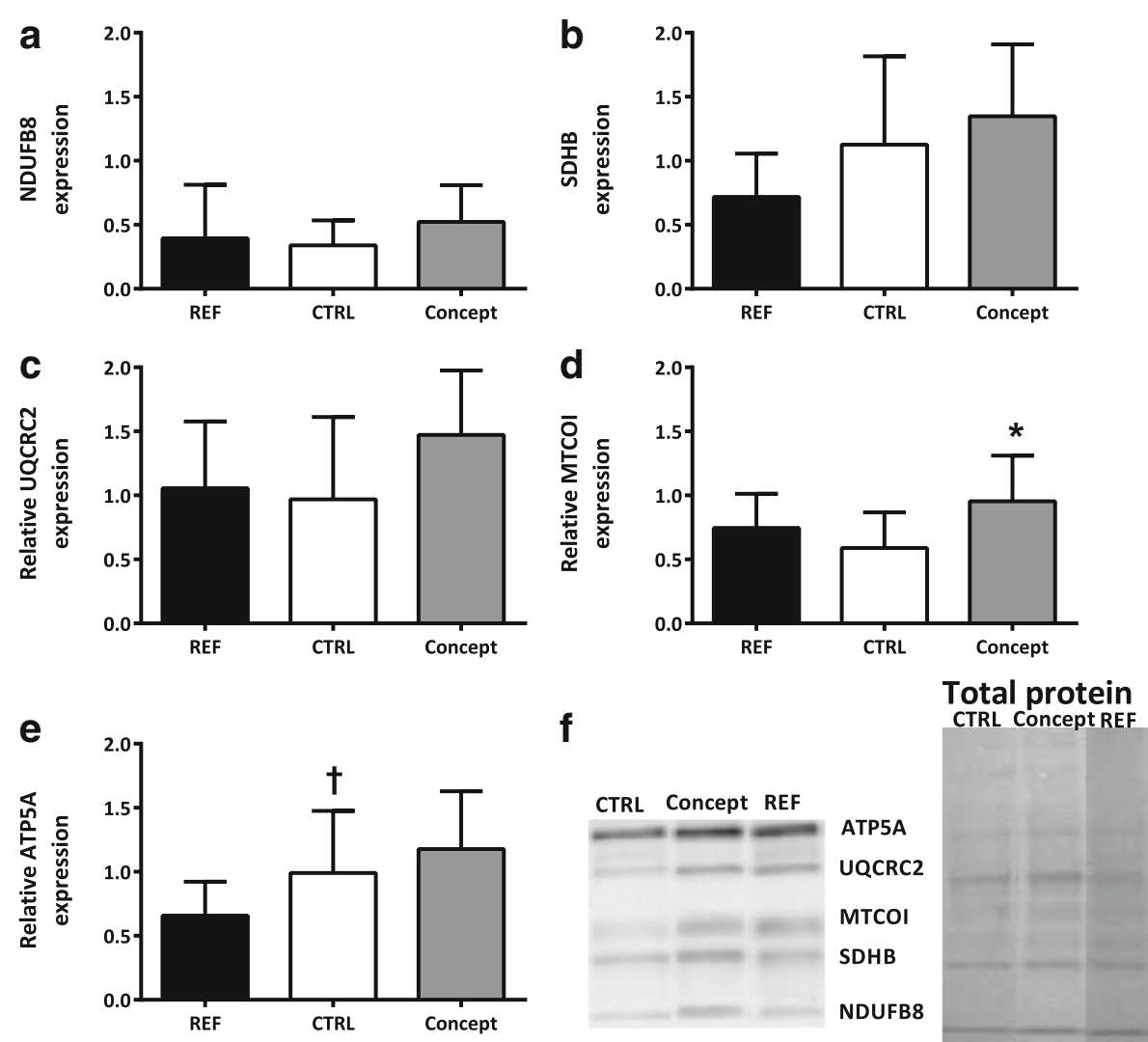

f
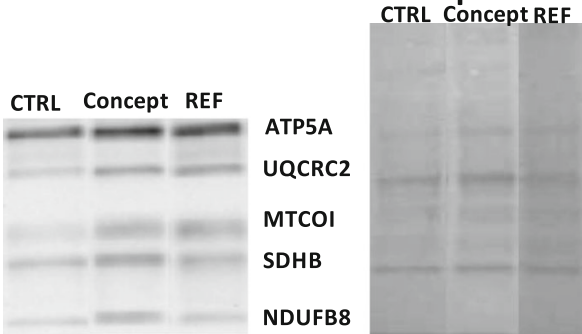

Fig. 4 Effect of postnatal Concept diet on relative protein expression of 5 oxidative phosphorylation complex (OXPHOS) subunits (a - e) in the retroperitoneal (RP) white adipose tissue ( $n=8$ for CTRL, $n=7$ for Concept and $n=10$ for REF group) and (f) total protein and western blot bands of one sample per group, samples came from one blot, but not from adjacent lanes, a picture of the whole blot is added as Additional file 1. Early diet and WSD challenge effects were analyzed separately. Difference between Concept and REF group not tested, as groups were fed different postnatal and adult diets. *: $p<0.05$, Concept different from CTRL group; +: $p$ 0.05-0.1 REF different from CTRL group

$L p l)$ and glucose transporter Glut4 were comparable between the Concept and the CTRL group (Table 4). Ppara (fat oxidative pathway) expression levels were also similar in the Concept and CTRL group as was the RP WAT expression of the uncoupling protein Ucp3 (Table 4). Expression of Ucp1, as marker for WAT browning, was similar between the Concept and CTRL group, but expression of another marker for WAT browning, Cidea tended to be higher in Concept compared to CTRL group $(p=0.08$; Table 4$)$.

\section{Gene expression profiles in skeletal muscle}

The Concept group showed higher Ucp3 expression levels in the $m$. tibialis compared to the CTRL group $(p<0.05$; Table 4). Expression profiles of Pdk4 (involved in the transport of pyruvate -the glycolytic end productinto the mitochondria), Cpt1 $\alpha$ (involved in the transport of long-chain fatty acids over the mitochondrial membrane) and Glut4 (glucose transporter) in the $m$. tibialis were not affected by the early postnatal diet (Table 4).

\section{Correlations}

Markers for mitochondrial content, CS activity and mtDNA, were inversely correlated to RP WAT weight $(r=-0.741 ; p<0.001$ and $r=-0.655 ; p<0.001$, respectively; Fig. 6). No correlations between protein expression of the OXPHOS complexes and RP WAT weight were found.

\section{Discussion}

The present study showed that early life exposure to large, phospholipid-coated lipid droplets leads to altered expression of markers for mitochondrial oxidative capacity in RP WAT and $m$. tibialis. Although functional mitochondrial capacity was not measured, these markers suggest that adapted mitochondrial oxidative capacity may underlie the previously reported reduced body fat accumulation in adolescence and adulthood [18-20].

Programming of metabolic health is well established, but possible underlying mechanisms are still largely unknown although many have been suggested. Aberrant mitochondrial function has been suggested in a limited amount of studies as possible link between adverse fetal 

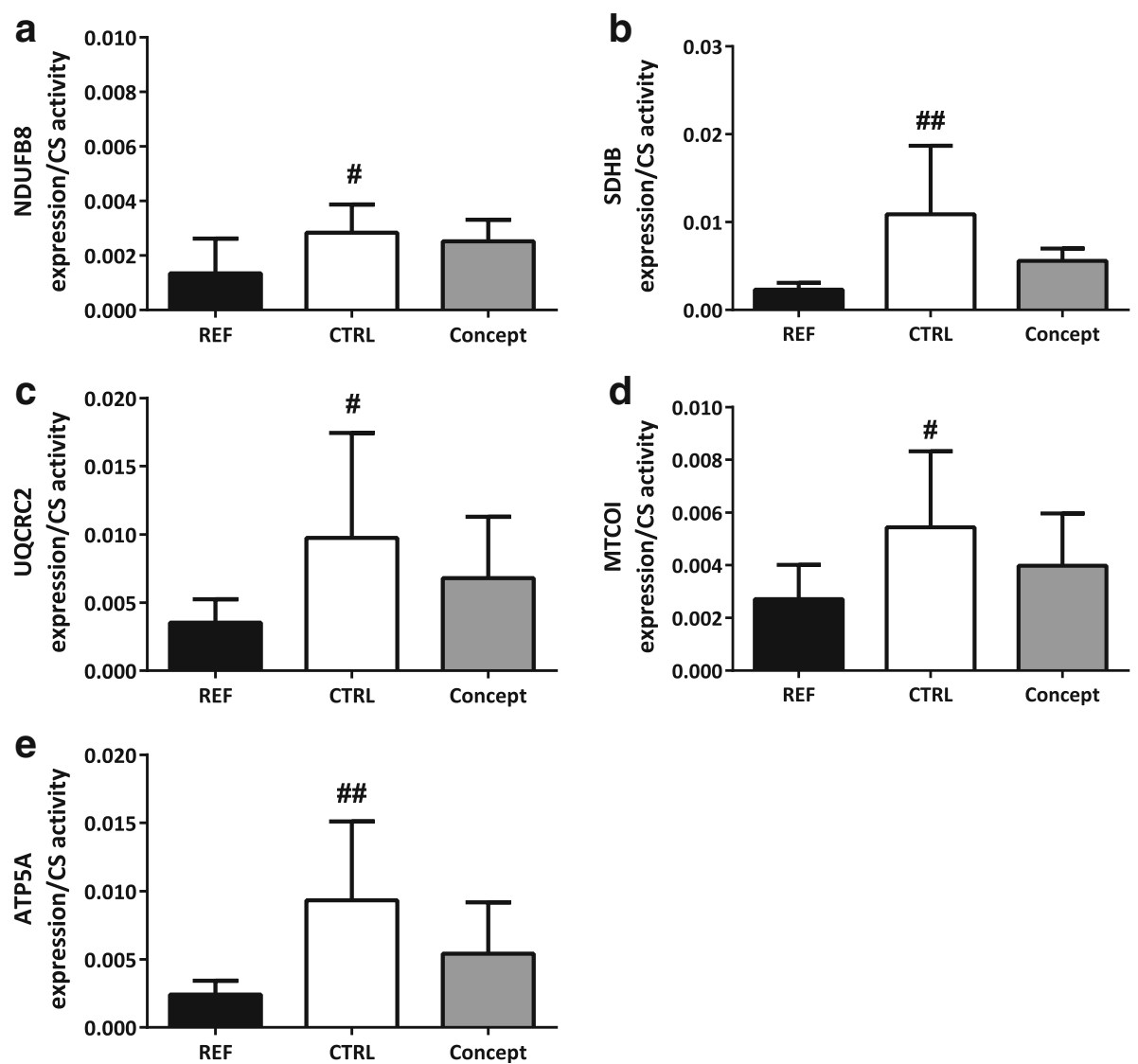

Fig. 5 Effect of postnatal Concept diet on relative protein expression of 5 oxidative phosphorylation complex (OXPHOS) subunits (a - e) in the retroperitoneal (RP) white adipose tissue when corrected for citrate synthase (CS) activity ( $n=7$ for CTRL, $n=6$ for Concept and $n=9$ for REF group). Early diet and WSD challenge effects were analyzed separately. Difference between Concept and REF group not tested, as groups were fed different postnatal and adult diets. \#: $p<0.05$; \#\#: $p<0.01$ different from CTRL group

environment and the development of T2D [22, 34]. Yet, little is known on how nutritional programming may improve lifelong metabolic health and protect against obesity.

In the present study, markers for mitochondrial oxidative capacity appeared to be increased due to the supramolecular structure of milk lipids in the early postnatal diet. The elevation in CS activity and relative mtDNA expression in the WAT imply higher mitochondrial content, suggesting a higher oxidative capacity. In addition, the elevated protein expression levels for the mitochondrial cytochrome $\mathrm{c}$ oxidase subunit I indicate that possibly more substrate is oxidized in RP WAT of Concept mice as well. As OXPHOS protein levels corrected for CS activity were similar between CTRL and Concept groups, total oxidative capacity per cell may be increased by the Concept diet rather than the intrinsic activity per mitochondrion. The expression levels of Ucp3 in the Concept group were slightly increased in skeletal muscle. A higher Ucp3 expression could elevate substrate oxidation via its uncoupling activity or via export of FA anions out of the mitochondria [35, 36]. Taken together, these data suggest that mitochondrial oxidative capacity may be elevated in mice fed a Concept diet in early postnatal life, resulting in a better ability to cope with an increased FA load in adulthood, which could explain the lower adiposity seen upon the WSD challenge.

The WSD challenge had, apart from the early diet, a clear effect on markers for oxidative capacity. Expression of markers for mitochondrial content (CS activity and relative mtDNA expression) in the WAT and mitochondrial uncoupling $(U c p 3)$ in the skeletal muscle were decreased in the CTRL compared to the non-challenge REF group. No effect of the WSD diet on OXPHOS protein levels was found. OXPHOS protein levels corrected for CS activity were however elevated in the CTRL compared to the REF group, which points towards increased intrinsic activity per mitochondrion as compensatory mechanism. These results are in line with the literature showing decreased expression of oxidative capacity markers in the WAT upon a high fat diet [37] and are opposite from the early diet effect. This may indicate 
Table 4 Relative mRNA expression in arbitrary units (AU)

\begin{tabular}{|c|c|c|c|}
\hline Gene name & $\operatorname{REF}(n=10)$ & $\operatorname{CTRL}(n=12)$ & Concept $(n=12)$ \\
\hline \multicolumn{4}{|l|}{ RP WAT: } \\
\hline Accl & $2.953(1.912-4.562)$ & $1.000(0.747-1.338){ }^{\# \# \#}$ & $1.212(0.815-1.803)$ \\
\hline Atgl & $1.264(1.118-1.429)$ & $1.000(0.786-1.273)+$ & $1.058(0.831-1.347)$ \\
\hline$C d 36$ & $0.863(0.741-1.006)$ & $1.000(0.939-1.064)^{\#}$ & $0.882(0.764-1.018) \neq$ \\
\hline Cidea & $10.066(6.317-16.038)$ & $1.000(0.603-1.659){ }^{\# \# \#}$ & $1.962(1.035-3.717) \neq$ \\
\hline Cpt1a & $0.711(0.595-0.849)$ & $1.000(0.816-1.225)^{\#}$ & $0.978(0.764-1.251)$ \\
\hline Fabp4 & $1.199(1.020-1.408)$ & $1.000(0.892-1.121)^{\#}$ & $1.097(1.002-1.200)$ \\
\hline Fas & $3.012(1.816-4.995)$ & $1.000(0.730-1.371)$ \# & $1.080(0.715-1.633)$ \\
\hline Fatp1 & $1.352(1.097-1.665)$ & $1.000(0.821-1.218)$ \# & $0.995(0.804-1.230)$ \\
\hline Glut4 & $1.939(1.315-2.858)$ & $1.000(0.863-1.159){ }^{\# \# \#}$ & $1.221(0.935-1.594)$ \\
\hline Gpat & $1.514(1.298-1.766)$ & $1.000(0.846-1.181))^{\# \# \# ~}$ & $1.167(0.925-1.472)$ \\
\hline$H k \|$ & $1.390(1.093-1.767)$ & $1.000(0.786-1.272)^{\#}$ & $1.068(0.795-1.434)$ \\
\hline$H s l$ & $1.146(1.048-1.252)$ & 1.000 (0.837-1.194) & $0.931(0.809-1.071)$ \\
\hline$L p l$ & $0.902(0.784-1.038)$ & $1.000(0.874-1.144)$ & $0.866(0.706-1.061)$ \\
\hline$P d k 4$ & $1.878(1.215-2.904)$ & $1.000(0.780-1.283){ }^{\# \#}$ & $1.377(0.887-2.140)$ \\
\hline Ppara & $1.911(1.648-2.216)$ & $1.000(0.785-1.274){ }^{\# \# \#}$ & $1.124(0.944-1.338)$ \\
\hline Scdl & $1.891(1.544-2.317)$ & $1.000(0.812-1.232){ }^{\# \# \#}$ & 1.079 (0.854-1.363) \\
\hline Srebplc & $1.426(1.146-1.775)$ & $1.000(0.810-1.235)$ \#\# & 1.087 (0.910-1.300) \\
\hline Ucp 1 & $27.480(15.828-47.709)$ & $1.000(0.337-2.968){ }^{\# \# \#}$ & $2.621(0.860-7.993)$ \\
\hline Ucp3 & $1.432(1.248-1.644)$ & $1.000(0.833-1.200){ }^{\# \#}$ & $1.168(0.883-1.544)$ \\
\hline Skeletal muscle: & $\operatorname{REF}(n=10)$ & $\mathrm{CTRL}(n=12)$ & Concept $(n=11)$ \\
\hline Cptia & $1.029(0.881-1.202)$ & $1.000(0.752-1.331)$ & $1.074(0.906-1.273)$ \\
\hline Glut4 & $1.009(0.811-1.256)$ & $1.000(0.811-1.233)$ & $1.089(0.939-1.262)$ \\
\hline$P d k 4$ & $1.600(1.124-2.277)$ & $1.000(0.684-1.461)$ & $1.296(0.929-1.808)$ \\
\hline Ucp3 & $1.577(1.286-1.933)$ & $1.000(0.830-1.204))^{\# \#}$ & $1.299(1.105-1.527)$ * \\
\hline
\end{tabular}

that mice fed the Concept diet in early life were protected against the adverse effects of the WSD challenge by preserving WAT health and were different from the CTRL mice, able to increase mitochondrial oxidative capacity in response to the WSD challenge.

Gene expression of markers for lipogenesis, lipolysis or lipid uptake were unaffected by the supramolecular lipid structure of the early postnatal diet, suggesting that these metabolic processes may not contribute the observed effects on adult adiposity. In addition, gene expression of markers for $\beta$-oxidation, transfer of longchain acyl-CoA into the mitochondria, other substrate transporters, the glucose oxidative pathway and browning of the WAT depot were not affected by the postnatal diet intervention.

It should be noted that functional measurements are pivotal to confirm whether the higher levels of mitochondrial content and capacity markers truly result in increased substrate oxidation upon a change in nutrient status. There is some evidence showing that mitochondrial function can be reduced or elevated upon caloric restriction and/or physical activity with similar numbers of mitochondria [38,39], indicating that similar numbers of mitochondria still may result in differences in mitochondrial function. In contrast, other studies have shown that mitochondrial content and complex IV protein content and activity were correlated to ADPstimulated respiration [40-42], suggesting that differences in levels of these markers may reflect an actual difference in mitochondrial function. Thus, although functional measurement were not performed, the lower adiposity and adipocyte size of the Concept group found, does suggest that the Concept mice showed a less exaggerated respond to the WSD challenge. This supports oxidation of the surplus of energy instead of storage into WAT.

Altered mitochondrial oxidative capacity in WAT could affect substrate selection and competition in other 


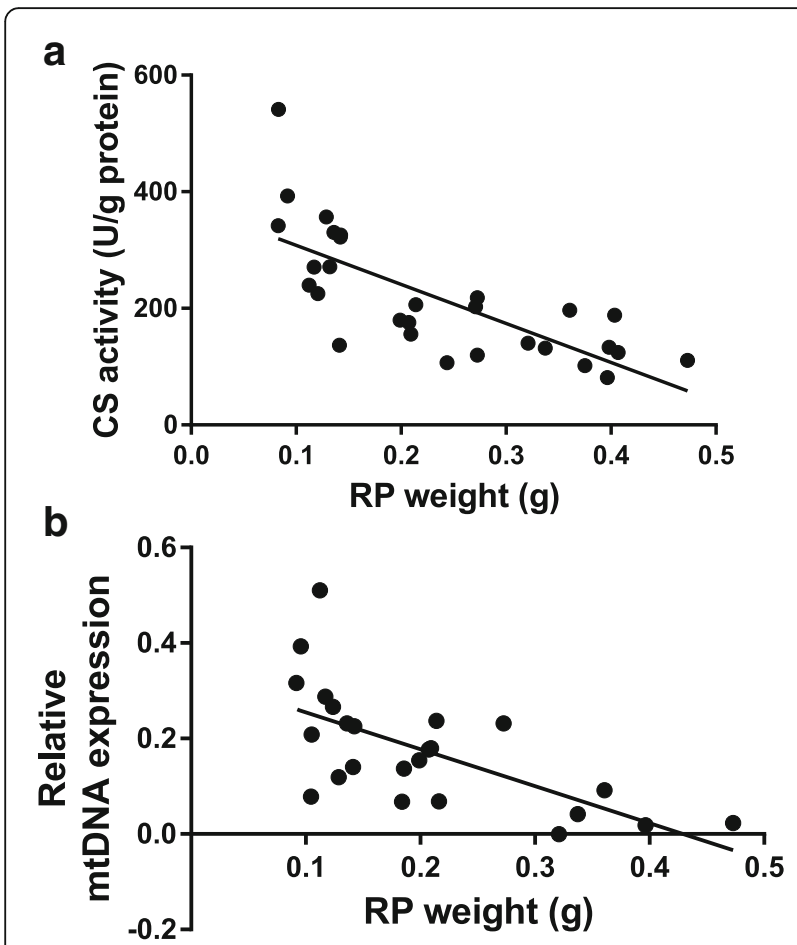

Fig. 6 Correlation between retroperitoneal (RP) white adipose tissue weight and (a) citrate synthase (CS) activity $(r=-0.741 ; p<0.001)$ and (b) mtDNA/nDNA ratio $(r=-0.655 ; p<0.001)$

oxidative organs like skeletal muscle and liver, thereby affecting whole body insulin sensitivity [43, 44]. Further studies focusing on functional measurements of mitochondrial function in liver and skeletal muscle are required to confirm that postnatal dietary lipid structure indeed programs adult mitochondrial function.

CS activity and mtDNA content, representative for mitochondrial content, were inversely correlated with RP WAT weight. Whether or not the higher mitochondrial content in the Concept group caused the reduced RP WAT weight or the other way around remains to be determined. Interestingly cytochrome c oxidase subunit I expression was not related to RP WAT weight, suggesting that OXPHOS protein expression may be driven by the interaction between the early diet and the WSD challenge although further investigation is needed to establish this.

Previous studies showed that mitochondrial oxidative capacity was decreased in rodents and sheep by exposure to HFD and/or malnutrition during fetal life [45-47]. The present results show a beneficial programming of mitochondrial oxidative capacity markers by a relatively mild diet intervention during the postnatal period. Based on these observations we could speculate that decreased oxidative capacity following an adverse fetal environment could potentially be reprogrammed by an adjusted diet in the postnatal period.

\section{Conclusion}

We showed that exposure to adapted supramolecular structure of dietary lipids (Nuturis ${ }^{\oplus}$ ) in early postnatal life results in increased expression of markers for mitochondrial content and capacity which may enable adult mice to cope better with the surplus of fat provided by the WSD challenge diet. Although functional measurements are not performed, these adaptations in mitochondrial function markers could underlie the observed reduced body fat accumulation in an obesogenic adult environment in these animals.

\section{Additional file}

Additional file 1: Figure S1. OXPHOS western blot. CTRL: sample of CTRL group; Conc: sample of Concept group; REF: sample of REF group; p.c.: positive control sample. (PDF 85 kb)

\section{Abbreviations}

18SrRNA: 18 S ribosomal RNA; Acc1: Acetyl-CoA carboxylase 1; AIN93G: American Institute of Nutrition-93G synthetic diet; Atgl: Adipose triglyceride lipase; ATP5A: ATP synthase subunit of complex $V$ of oxidative phosphorylation; Canx: Calnexin; Cd36: Fatty acid transfer protein/cluster of differentiation 36; Cpt1a: Carnitinepalmitoyl-transferase 1a; CS: Citrate synthase; EPI: Epididymal fat depot; FA: Fatty acids; Fabp4: Fatty acid binding protein 4; Fasn: Fatty acid synthase; Fatp 1: Fatty acid transfer protein 1; Glut4: Glucose transporter 4; Gpat: Glycerol-3-phosphate acyltransferase; HAD: Hydroxyacyl-CoA dehydrogenase; HFD: High fat diet; Hkll: Hexokinase II; Hsl: Hormone sensitive lipase; IMF: Infant milk formula; Lpl: Lipoprotein lipase; MTCOI: Mitochondrial encoded cytochrome C oxidase I subunit of complex IV of oxidative phosphorylation; MtDNA: Mitochondrial DNA; Nd1: NADH dehydrogenase 1; NDNA: Nuclear DNA; NDUFB8: NADH dehydrogenase 1 beta subunit of complex I of oxidative phosphorylation; OXPHOS: Oxidative phosphorylation complex; Pdk4: Pyruvate dehydrogenase kinase 4; PN: Postnatal day; Ppara: Peroxisome proliferator activated receptor a; RP: Retroperitoneal fat depot; Rp/19: Ribosomal protein L19; Rps29: Ribosomal protein S29; Scd1: Stearoyl-Coenzyme A desaturase 1; SDHB: Succinate dehydrogenase subunit of complex II of oxidative phosphorylation: Srebp1c: Sterol regulatory element binding protein 1C; T2D: Type 2 diabetes; UCp: Uncoupling protein; UQCRC2: Ubiquinol cytochrome $C$ reductase core protein subunit III of oxidative phosphorylation; WAT: White adipose tissue; WSD: Western style diet

\section{Acknowledgements}

We thank Diane Kegler for help in performing the animal experiment and Annemarie Baars for skeletal muscle gene expression analyses.

\section{Funding}

Nutricia Reaearch funded the study.

\section{Availability of data and materials}

The datasets generated during the current study are available from the corresponding author on reasonable request.

\section{Authors' contributions}

$A K, A O, E P, E E$, LS and EvdB design of the study; AK, EE, LS, performed experiments, $A K$ analyzed data; $A K, A O, E P, E v d B$ interpreted results; $A K$ prepared figures; $A K, A O, E P$ and EvdB drafted manuscript; $A K, A O, E P, E E, L S$, EvdB edited and revised manuscript; $A K, A O, E P, E E, L S, E v d B$ approved the final version of manuscript.

\section{Competing interests}

Andrea Kodde, Annemarie Oosting, Eefje Engels, Lidewij Schipper and Eline van der Beek are employees of Nutricia Research. 


\section{Consent for publication}

Not applicable.

\section{Ethics approval and consent to participate}

All experimental procedures were approved by an external, independent Animal Experimental Committee (DEC consult, Soest, The Netherlands) and complied with the principles of good laboratory animal care following the EU-directive for the protection of animals used for scientific purposes.

\section{Author details}

'Earl Life Nutrition Division, Nutricia Research, P.O. Box 801413508 TC Utrecht, The Netherlands. 'Department of Pediatrics, University Medical Centre Groningen, Groningen, The Netherlands. ${ }^{3}$ Department of Human Biology, Maastricht University, Maastricht, The Netherlands.

Received: 8 November 2016 Accepted: 31 May 2017 Published online: 12 June 2017

\section{References}

1. Lobstein T, Baur L, Uauy R. Obesity in children and young people: a crisis in public health. Obes Rev. 2004;5(Suppl 1):4-104

2. Rennie $K L$, Johnson $L$, Jebb SA. Behavioural determinants of obesity. Best Pract Res Clin Endocrinol Metab. 2005;19:343-58.

3. Spalding KL, Arner E, Westermark PO, Bernard S, Buchholz BA, Bergmann O, et al. Dynamics of fat cell turnover in humans. Nature. 2008;453:783-7.

4. Surwit RS, Feinglos MN, Rodin J, Sutherland A, Petro AE, Opara EC, et al. Differential effects of fat and sucrose on the development of obesity and diabetes in C57BL/6J and a/J mice. Metabolism. 1995:44:645-51.

5. Berger JJ, Barnard RJ. Effect of diet on fat cell size and hormone-sensitive lipase activity. J Appl Physiol. 1999;87:227-32.

6. Kahn BB, Flier JS. Obesity and insulin resistance. J Clin Invest. 2000;106:473-81.

7. Rogge MM. The role of impaired mitochondrial lipid oxidation in obesity. Biol Res Nurs. 2009;10:356-73.

8. Phielix E, Mensink M. Type 2 diabetes mellitus and skeletal muscle metabolic function. Physiol Behav. 2008;94:252-8.

9. Kelley DE, He J, Menshikova EV, Ritov VB. Dysfunction of mitochondria in human skeletal muscle in type 2 diabetes. Diabetes. 2002;51:2944-50.

10. Mogensen M, Sahlin K, Fernstrom M, Glintborg D, Vind BF, Beck-Nielsen $H_{\text {, }}$ et al. Mitochondrial respiration is decreased in skeletal muscle of patients with type 2 diabetes. Diabetes. 2007:56:1592-9.

11. Wilson-Fritch L, Nicoloro S, Chouinard M, Lazar MA, Chui PC, Leszyk J, et al Mitochondrial remodeling in adipose tissue associated with obesity and treatment with rosiglitazone. J Clin Invest. 2004;114:1281-9.

12. Holmstrom MH, Iglesias-Gutierrez E, Zierath JR, Garcia-Roves PM. Tissuespecific control of mitochondrial respiration in obesity-related insulin resistance and diabetes. Am J Physiol Endocrinol Metab. 2012;302:E731-9.

13. Rong JX, Qiu Y, Hansen MK, Zhu L, Zhang V, Xie M, et al. Adipose mitochondrial biogenesis is suppressed in $\mathrm{db} / \mathrm{db}$ and high-fat diet-fed mice and improved by rosiglitazone. Diabetes. 2007;56:1751-60.

14. Bayol SA, Simbi BH, Bertrand JA, Stickland NC. Offspring from mothers fed a 'junk food' diet in pregnancy and lactation exhibit exacerbated adiposity that is more pronounced in females. J Physiol. 2008;586:3219-30.

15. Turdi S, Ge W, Hu N, Bradley KM, Wang X, Ren J. Interaction between maternal and postnatal high fat diet leads to a greater risk of myocardial dysfunction in offspring via enhanced lipotoxicity, IRS-1 serine phosphorylation and mitochondrial defects. J Mol Cell Cardiol. 2013:55:117-29.

16. Harder T, Bergmann R, Kallischnigg G, Plagemann A. Duration of breastfeeding and risk of overweight: a meta-analysis. Am J Epidemiol. 2005;162:397-403.

17. Owen CG, Martin RM, Whincup PH, Smith GD, Cook DG. Effect of infant feeding on the risk of obesity across the life course: a quantitative review of published evidence. Pediatrics. 2005;115:1367-77.

18. Oosting A, Kegler D, Wopereis HJ, Teller IC, van de Heijning BJ, Verkade HJ, et al. Size and phospholipid coating of lipid droplets in the diet of young mice modify body fat accumulation in adulthood. Pediatr Res. 2012;72:362-9.

19. Oosting A, van Vlies N, Kegler D, Schipper L, Abrahamse-Berkeveld M, Ringler $\mathrm{S}$, et al. Effect of dietary lipid structure in early postnatal life on mouse adipose tissue development and function in adulthood. $\mathrm{Br} J$ Nutr. 2013:1-12.

20. Baars A, Oosting A, Engels E, Kegler D, Kodde A, Schipper L, et al. Milk fat globule membrane coating of large lipid droplets in the diet of young mice prevents body fat accumulation in adulthood. Br J Nutr. 2016:1-8.

21. Bruce KD, Cagampang FR, Argenton M, Zhang J, Ethirajan PL, Burdge GC, et al. Maternal high-fat feeding primes steatohepatitis in adult mice offspring, involving mitochondrial dysfunction and altered lipogenesis gene expression. Hepatology. 2009;50:1796-808.

22. Taylor PD, McConnell J, Khan IY, Holemans K, Lawrence KM, Asare-Anane H, et al. Impaired glucose homeostasis and mitochondrial abnormalities in offspring of rats fed a fat-rich diet in pregnancy. Am J Phys Regul Integr Comp Phys. 2005;288:R134-9.

23. Shelley P, Martin-Gronert MS, Rowlerson A, Poston L, Heales SJ, Hargreaves $I P$, et al. Altered skeletal muscle insulin signaling and mitochondrial complex II-III linked activity in adult offspring of obese mice. Am J Phys Regul Integr Comp Phys. 2009;297:R675-81.

24. Simmons RA, Suponitsky-Kroyter I, Selak MA. Progressive accumulation of mitochondrial DNA mutations and decline in mitochondrial function lead to beta-cell failure. J Biol Chem. 2005;280:28785-91.

25. Reeves PG, Nielsen FH, Fahey GC Jr. AIN-93 purified diets for laboratory rodents: final report of the American Institute of Nutrition ad hoc writing committee on the reformulation of the AIN-76A rodent diet. J Nutr. 1993;123:1939-51.

26. Gallier S, Vocking K, Post JA, Van De Heijning B, Acton D, Van Der Beek EM, et al. A novel infant milk formula concept: mimicking the human milk fat globule structure. Colloids Surf B: Biointerfaces. 2015;136:329-39.

27. DiGirolamo M, Fine JB. Cellularity measurements. Methods Mol Biol. 2001; 155:65-75.

28. Hirsch J, Gallian E. Methods for the determination of adipose cell size in man and animals. J Lipid Res. 1968;9:110-9.

29. Vanhoutvin SA, Troost FJ, Hamer HM, Lindsey PJ, Koek GH, Jonkers DM, et al. Butyrate-induced transcriptional changes in human colonic mucosa. PLoS One. 2009:4:e6759.

30. Hellemans J, Mortier G, De Paepe A, Speleman F, Vandesompele J. qBase relative quantification framework and software for management and automated analysis of real-time quantitative PCR data. Genome Biol. 2007;8:R19.

31. Shepherd D, Garland PB. The kinetic properties of citrate synthase from rat liver mitochondria. Biochem J. 1969;114:597-610.

32. Meex RC, Phielix E, Schrauwen-Hinderling V, Moonen-Kornips E, Schaart G, Schrauwen $P$, et al. The use of statins potentiates the insulin-sensitizing effect of exercise training in obese males with and without type 2 diabetes. Clin Sci. 2010;119:293-301.

33. Kaaman M, Sparks LM, van Harmelen V, Smith SR, Sjolin E, Dahlman I, et al. Strong association between mitochondrial DNA copy number and lipogenesis in human white adipose tissue. Diabetologia. 2007;50:2526-33.

34. Warner MJ, Ozanne SE. Mechanisms involved in the developmental programming of adulthood disease. Biochem J. 427:333-47.

35. Schrauwen P, Hoeks J, Schaart G, Kornips E, Binas B, Van De Vusse GJ, et al. Uncoupling protein 3 as a mitochondrial fatty acid anion exporter. FASEB J. 2003;17:2272-4.

36. Brand MD, Pamplona R, Portero-Otin M, Requena JR, Roebuck SJ, Buckingham JA, et al. Oxidative damage and phospholipid fatty acyl composition in skeletal muscle mitochondria from mice underexpressing or overexpressing uncoupling protein 3. Biochem J. 2002;368:597-603.

37. Cummins TD, Holden CR, Sansbury BE, Gibb AA, Shah J, Zafar N, et al. Metabolic remodeling of white adipose tissue in obesity. Am J Physiol Endocrinol Metab. 2014;307:E262-77.

38. Rabol R, Svendsen PF, Skovbro M, Boushel R, Haugaard SB, Schjerling P, et al. Reduced skeletal muscle mitochondrial respiration and improved glucose metabolism in nondiabetic obese women during a very low calorie dietary intervention leading to rapid weight loss. Metabolism. 2009;58:1 145-52.

39. Menshikova EV, Ritov VB, Toledo FG, Ferrell RE, Goodpaster BH, Kelley DE. Effects of weight loss and physical activity on skeletal muscle mitochondrial function in obesity. Am J Physiol Endocrinol Metab. 2005;288:E818-25.

40. Larsen S, Nielsen J, Hansen CN, Nielsen LB, Wibrand F, Stride N, et al. Biomarkers of mitochondrial content in skeletal muscle of healthy young human subjects. J Physiol. 2012;590:3349-60.

41. Kraunsoe R, Boushel R, Hansen CN, Schjerling P, Qvortrup K, Stockel M, et al. Mitochondrial respiration in subcutaneous and visceral adipose tissue from patients with morbid obesity. J Physiol. 2010;588:2023-32. 
42. Deveaud C, Beauvoit B, Salin B, Schaeffer J, Rigoulet M. Regional differences in oxidative capacity of rat white adipose tissue are linked to the mitochondrial content of mature adipocytes. Mol Cell Biochem. 2004:267:157-66.

43. Vernochet C, Damilano F, Mourier A, Bezy O, Mori MA, Smyth G, et al. Adipose tissue mitochondrial dysfunction triggers a lipodystrophic syndrome with insulin resistance, hepatosteatosis, and cardiovascular complications. FASEB J. 2014;28:4408-19.

44. Choo HJ, Kim JH, Kwon OB, Lee CS, Mun JY, Han SS, et al. Mitochondria are impaired in the adipocytes of type 2 diabetic mice. Diabetologia. 2006:49:784-91.

45. Jorgensen W, Gam C, Andersen JL, Schjerling P, Scheibye-Knudsen M, Mortensen $\mathrm{OH}$, et al. Changed mitochondrial function by pre- and/or postpartum diet alterations in sheep. Am J Physiol Endocrinol Metab. 2009;297:E1349-57.

46. Latouche C, Heywood SE, Henry SL, Ziemann M, Lazarus R, El-Osta A, et al. Maternal overnutrition programs changes in the expression of skeletal muscle genes that are associated with insulin resistance and defects of oxidative phosphorylation in adult male rat offspring. J Nutr. 2014;144:23744

47. Park KS, Kim SK, Kim MS, Cho EY, Lee JH, Lee KU, et al. Fetal and early postnatal protein malnutrition cause long-term changes in rat liver and muscle mitochondria. J Nutr. 2003;133:3085-90.

\section{Submit your next manuscript to BioMed Central and we will help you at every step:}

- We accept pre-submission inquiries

- Our selector tool helps you to find the most relevant journal

- We provide round the clock customer support

- Convenient online submission

- Thorough peer review

- Inclusion in PubMed and all major indexing services

- Maximum visibility for your research

Submit your manuscript at www.biomedcentral.com/submit 